\title{
Middle-aged mothers live longer
}

Comparing two groups of women born Uin 1896, we found that women who lived to at least age 100 were four times more likely to have had children while in their forties than women who survived only to age 73 . The ability to have children in the fifth decade may be a marker for slow ageing and subsequent ability to achieve extreme longevity. We propose that the evolutionary pressure to extend lifespan is closely linked to prolonging the period of time during which women can bear children.

We compared a group of 78 female centenarians living in the suburban Boston area (Massachusetts, USA) with a similar birth cohort of 54 women born in 1896, but who died at 73 years of age in 1969 (Fig. 1). We located the next-of-kin of these subjects using data provided by the Massachusetts Registry of Vital Records. By comparing women with the same year of birth, we minimized concerns about temporally related influences upon fertility such as health and contraception-related trends, war, fluctuations in the economy (the boom of the 1920s, the Great Depression) and so on. Subjects from both the centenarian and septuagenarian groups were excluded if they did not have the opportunity to have children (never married or hysterectomy before age 35 years). There were no significant differences between the two cohorts in terms of rate of hysterectomy, loss of spouse before age 45 , marriage without children, years of education, religion or race.

Our findings may allow the prediction of individuals predisposed to extreme longevity and may also have implications regarding the theoretical basis of menopause and human lifespan. During the first quarter of this century, fertility-enhancing interventions for older women were not available. Under these circumstances, later menopause, as well as pregnancy after age 40, may be associated with extreme longevity.

Menopause could act evolutionarily to protect the ageing woman from the hazards

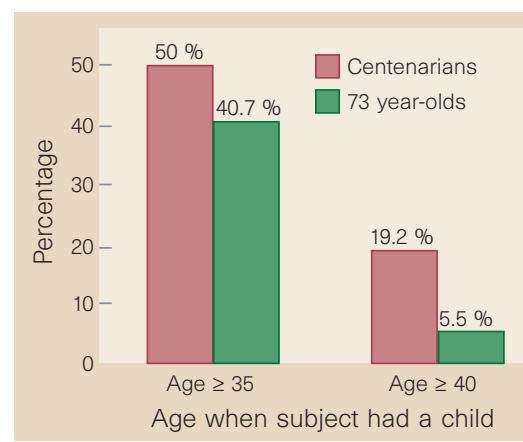

Figure 1 Percentage of women having children at or beyond ages 35 and 40 . The difference in the number giving birth at or after age 35 in the two groups was not statistically significant (odds ratio = 1.5, 95\% confidence interval $(\mathrm{Cl})$ : 0.7-3.1, $P=0.3)$, but was significant in those giving birth at or after age 40 (odds ratio $=4.0,95 \% \mathrm{Cl}: 1.02-18.7, P=0.02$ ).

of childbirth. We therefore support the theory that as humans evolved and became able to reach greater ages, there came a point when survival during childbirth began to decline as a function of further ageing and increased frailty ${ }^{1}$. 'Premature' death of the mother would also put at risk any existing children and their potential for reproduction ${ }^{2}$. At the age when the risk of child-bearing outweighs the benefit of producing progeny, natural selection would favour women who became infertile; thus, the evolutionary pressure for menopause.

Although menopause still occurs, the genes that allowed a woman to age more slowly continue to exert their influence. A continued slow rate of ageing and perhaps also a decreased susceptibility to diseases associated with ageing that can cause premature mortality would therefore allow a woman to achieve extreme longevity. These observations in humans concur with selection experiments in fruitflies in which the ability to produce eggs later in life is correlated with greater life expectancy ${ }^{3}$.

It was recently noted that women who took oestrogen were less likely to develop
Alzheimer's disease or were likely to show a delay in onset if the disease did develop ${ }^{4}$. The same may be true for women who go through menopause later, who have a prolonged exposure to endogenously produced oestrogen (or other concomitantly produced substances). By avoiding, or at least delaying, diseases associated with ageing that can cause premature mortality, such as Alzheimer's disease, heart disease or stroke, these women can therefore achieve greater longevity.

It has been suggested that the selective forces determining longevity in females were the driving force behind the lifespan of humans ${ }^{5}$. Extending this theory, we argue that the driving selective force of human lifespan is maximizing the period of time during which women can bear children. The age at which female survival is diminished by further reproduction may therefore be the determinant of subsequent lifespan. One might ask why menopause does not occur in most other mammalian species ${ }^{6,7}$, and the answer may be that in those species the mortality risk of giving birth is relatively low even at advanced age and does not outweigh the benefit. We should now search for associations between genes that regulate reproductive fitness and ovarian ageing and genes that regulate rates of ageing and susceptibility to diseases associated with ageing.

Thomas T. Perls ${ }^{\star}$, Laura Alpert ${ }^{\star}$

Ruth C. Fretts $\dagger$

${ }^{*}$ Gerontology Division, Department of Medicine and $†$ Department of Obstetrics and Gynecology,

Beth Israel-Deaconess Medical Center,

Harvard Medical School, Boston,

Massachusetts 02215, USA

e-mail: tperls@warren.med.harvard.edu

\footnotetext{
1. Williams, G. C. \& Williams, D. Evolution 11, 398-411 (1957).

2. Rochat, R. W et al. Obstet. Gynecol. 72, 91-97 (1988).

3. Hutchinson, E. W., Shaw, A. J. \& Rose, M. R. Genetics 127, 729-737 (1991).

4. Tang, M. X. et al. Lancet 348, 429-432 (1996).

5. Holliday, R. Perspect. Biol. Med. 40, 100-107 (1996).

6. Gould, K. G., Flint, M. \& Graham, C. E. Maturitas 3, 157-166 (1981)

7. Austad, S. N. Exp. Gerontol. 29, 255-263 (1994).
}

\section{Has BCG attenuated}

\section{to impotence?}

The magnitude of the global tuberculosis problem and the emergence of antibioticresistant organisms has resulted in a renewed need for tuberculosis vaccine development. An ideal vaccine would consistently confer protection without confounding the interpretation of the tuberculin skin test. The current vaccine, Bacille Calmette-Guérin (BCG), provides inconsistent efficacy $(0-80 \%$ in randomized control trials) yet usually induces tuberculin reactivity. This variability in observed efficacy has been attributed to differences in trial methodology, host genetics and immunity to a variety of environmental mycobacteria $^{1}$. We hypothesize that the attributes of the current BCG have resulted from subtle pressures to minimize adverse reactions and maintain tuberculin reactivity during vaccine development and testing.

For BCG strains used in more than one clinical trial, we plotted efficacy and induced tuberculin reactivity against in vitro passage number. Four out of five strains have apparently lost efficacy while maintaining tuberculin reactivity (Fig. 1). Considering that attenuation during 230 in vitro passages was used by Calmette and Guérin to produce their vaccine, it is not surprising that protective efficacy of BCG waned over the next 1,000 passages. In 1921 Calmette declared this vaccine a "virus fixé" , but there is ample evidence that this live bacterial vaccine is far from fixed, with daughter strains differing in mycolic acid composition, protein transcription and genotype ${ }^{1}$. Over the course of this evolution 

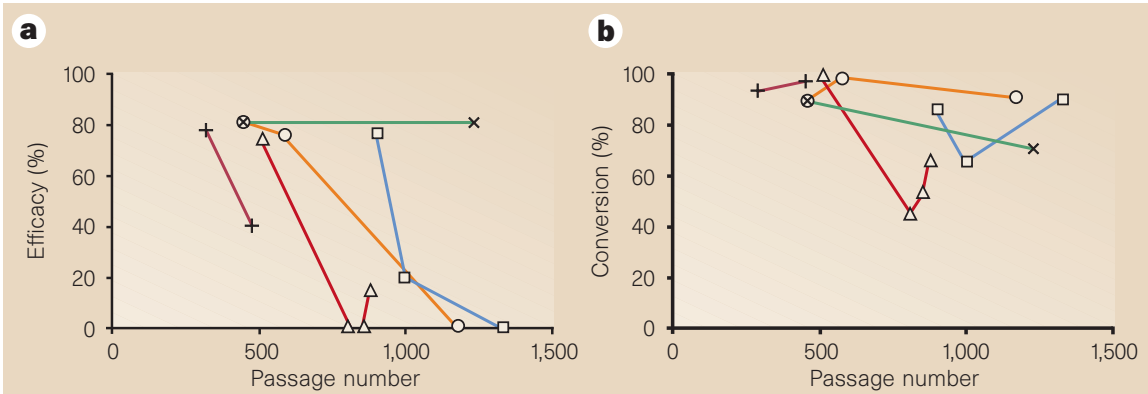

Figure 1 Efficacy (a) and tuberculin conversion rates (b) of BCG substrains plotted against in vitro passage number. Strains: Pasteur (s ), Phipps (+), Denmark (u ), Tice (n) and Frappier ( $\times$ ). Estimates of efficacy and tuberculin reactivity are from a recent meta-analysis of $\mathrm{BCG}^{9}$ and a recent review ${ }^{1}$, with the exception that results in a North American Indian trial have been separated by villages receiving BCG-Pasteur and BCGPhipps ${ }^{10}$. Estimates less than zero are plotted as $0 \%$ because confidence limits were large and spanned zero. Passage number of strains are those reported in the studies; if not specified, passage number was interpolated from data before and after the trial.

of BCG strains, there was a secular trend towards decreasing tuberculosis mortality, so public acceptance of adverse effects diminished.

Meanwhile, a selective pressure to maintain tuberculin reactivity resulted from the misperception that it served as a proxy for protection and the fact that it could be readily assessed. Thus, studies were performed with the goal of decreasing adverse reactions while retaining tuberculin conver$\operatorname{sion}^{3}$. More recent work has shown that tuberculin conversion and protective immunity can be dissociated ${ }^{4}$, and that virulence appears to correlate with protective efficacy in animal models ${ }^{5,6}$. This trade-off had already been noted in 1952 by Rene Dubos who wrote, "Very properly, the greatest emphasis is always placed on administering the vaccine in such a manner that no unpleasant reaction ensues, but because vaccination must be safe for all, it is probably ineffective for many..." . The detailed manner in which these selective pressures affected vaccine development cannot be deduced from the historical record, but it is noteworthy that by 1956 , half of international vaccine producers $(20$ of 40) had changed strain at some point ${ }^{8}$.

There are three important implications of this hypothesis. First, estimates of BCG efficacy using meta-analyses may significantly overestimate current efficacy by averaging the effects of current and ancestral vaccines. An accurate assessment of currently available strains is essential in the design of rational vaccine policy. Second, comparison within strains of recent and more ancestral forms (such as retention lots from vaccine trials) may provide insight into vaccine characteristics that correlate with efficacy and tuberculin conversion. Resulting insights may be exploited to increase protection and decrease tuberculin conversion rates of new vaccines. Third, in the absence of increased understanding of the fundamental determinants of virulence and protection, it is likely that increased efficacy will be achieved only at the cost of increased adverse reactions.

Marcel A. Behr, Peter M. Small

Division of Infectious Diseases

and Geographic Medicine,

Stanford University Medical Center,

300 Pasteur Drive, Stanford,

California 94305-5107, USA

e-mail:marcel@molepi.stanford.edu

1. Comstock, G. W. Control Clin. Trials 15, 247-276 (1994).

2. Calmette, A. Proc. R. Soc. Med. 24, 85-94 (1931).

3. Edwards, L. B. \& Gelting, A. S. Bull. World Health Org. 3, 279-300 (1950).

4. Orme, I. M. \& Collins, F. M. Cell. Immunol. 84, 113-120 (1984).

5. Dubos, R. J. \& Pierce, C. H. Am. Rev. Tuberculosis 74, 699-717 (1948).

6. Jespersen, A. \& Bentzon, M. W. Acta Tuberculosis Pneumol. Scand. 44, 253-289 (1964).

7. Dubos, R. \& Dubos, J. The White Plague: Tuberculosis, Man and Society 163 (Little, Brown \& Co., Boston, 1952).

8. Frappier, A. \& Panisset, M. La Souche du BCG 93 (Institut de Microbiol. et d'Hygiene, Univ. Montreal, Montreal, Canada, 1957).

9. Colditz, G. A. et al. J. Am. Med. Assoc. 271, 698-702 (1994).

10. Aronson, J. D. Am. Rev. Tuberculosis 58, 255-281 (1948).

\section{Inhibition of

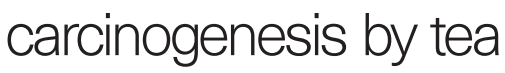

In the recent Scientific Correspondence ${ }^{1}$ by J. Jankun et al., entitled "Why drinking tea could prevent cancer", I was surprised to find that a review article by myself and Z.-Y. Wang about the effects of green and black tea on cancer ${ }^{2}$ had been misquoted. In addition, I find the hypothesis in this report as to why drinking tea could prevent cancer misleading, and believe that the data were erroneously interpreted with regard to the mechanisms of cancer inhibition by tea. I would like to clarify four points.

First, Jankun et al. wrote that "breast and prostate cancers in animal models are reduced by green, but not black, tea"1 ${ }^{\text {, citing }}$ my review ${ }^{2}$. But the review provides no evidence to support this statement. The review discussed the inhibitory activities of both green and black tea in animal models. In the lung tumorigenesis model with mice and the oesophageal tumorigenesis model with rats, green and black tea had similar inhibitory activities ${ }^{3-5}$. Black tea can inhibit the hyperproliferation of lung epithelial cells and the progression from adenoma to adenocarcinoma in the mouse lung ${ }^{6}$. There is no evidence from the review or elsewhere to support the statement that "the brewing of black tea oxidizes the cathechins, destroying any beneficial effects"1. Although the inhibitory activities of green and black tea have been demonstrated convincingly in animal studies, such activity has been shown in some, but not other, epidemiological studies $^{2,7}$. The cancer-preventing effect of tea in humans requires further study.

Second, my laboratory has conducted several studies indicating that the blood level of epigallocatechin-3-gallate (EGCG) after consuming the equivalent of $2-3$ cups of tea was $0.1-0.6 \mu \mathrm{M}$ and for an equivalent of 7-9 cups was still lower than $1 \mu \mathrm{M}^{8-10}$. In studies with mice and rats in which inhibition of skin, lung and oesophageal tumorigenesis was found, the blood EGCG level was $0.1-0.3 \mu \mathrm{M}$ and the tissue (such as the lung) levels were very low - the highest tissue level, close to $1 \mu \mathrm{M}$, was observed in the oesophageal epithelia ${ }^{9}$. The effective concentration needed to inhibit urokinase (2-10 mM), as reported by Jankun et al. ${ }^{1}$, was at least 3 or 4 orders of magnitude higher than the expected tissue levels. Given this information, how could inhibition of urokinase be related to cancer prevention in animal models and in humans?

Third, we and others have shown that micromolar concentrations of EGCG, other tea cathechins, and theaflavins (a characteristic group of compounds in black tea) inhibited the cell growth of many human cancer and other cell lines. Even in these cases, it cannot be concluded that this activity is related to the inhibition of tumorigenesis. EGCG binds strongly to many biological molecules and affects a variety of enzyme activities and signal transduction pathways at micromolar or even nanomolar concentrations ${ }^{2,9}$. Thus I believe that inhibition of urokinase at millimolar concentrations is unlikely to be a viable mechanism for the inhibition of tumorigenesis.

Fourth, as discussed in my review and in other publications ${ }^{2,9}$, the inhibitory activity of tea against tumorigenesis is not due to a single compound, but rather the combined activities of several or many constituents of tea. The mechanisms of action of tea need to be further elucidated before sweeping conclusions can be drawn.

\section{Chung S. Yang}

Laboratory for Cancer Research, Department of Chemical Biology,

College of Pharmacy, Frelinghuysen Road, Piscataway, New Jersey 08855-0789, USA e-mail: csyang@rci.rutgers.edu 